\title{
Comparison of Good Medical Practice Guidelines of Malaysia, United Kingdom and Australia: A Review Paper
}

\author{
Hanusha Durganaudu, Goh Wern Sze, Yan Seah Yuin, Naganathan Pillai, Christina Gertrude Yap, \\ Nowrozy Kamar Jahan*
}

Jeffrey Cheah School of Medicine and Health Sciences, Monash University Malaysia, Selangor Darul Ehsan, Malaysia

Email: *nowrozy.jahan@monash.edu

How to cite this paper: Durganaudu, H., Sze, G.W., Yuin, Y.S., Pillai, N., Yap, C.G. and Jahan, N.K. (2021) Comparison of Good Medical Practice Guidelines of Malaysia, United Kingdom and Australia: A Review Paper. Open Access Library Journal, 8: e7546.

https://doi.org/10.4236/oalib.1107546

Received: May 19, 2021

Accepted: June 25, 2021

Published: June 28, 2021

Copyright () 2021 by author(s) and Open Access Library Inc.

This work is licensed under the Creative Commons Attribution International License (CC BY 4.0).

http://creativecommons.org/licenses/by/4.0/

(c) (i) Open Access

\begin{abstract}
Good medical practice guidelines primarily highlight the obligations expected from medical practitioners, which are considered safe, effective and trustworthy by practitioners and the general community. Hence, the purpose of these guidelines is to serve as guidance for doctors to provide the best quality of care for their patients. While the core themes incorporated in these guidelines are often similar, there is nevertheless a need to review the existing good medical practice guidelines to determine the key themes, similarities, differences and gaps across different countries. This review has thoroughly explored the most recently published good medical practice guidelines from three countries, namely the United Kingdom, Australia, and Malaysia. Extracted information was classified under four different domains. Sub-domains were generated based on the general ideas and concepts discussed under each domain. We found a significant number of similarities and overlaps among the three guidelines; however, it is worth pointing out that variations exist in terms of sub-domains and depth of discussions for particular aspects as well. This review serves as a comprehensive compilation of good medical practice guidelines for medical students and doctors to aid their daily practice. Moreover, the findings in this review facilitates the policy-makers as a guide while subsequently updating the good medical practice guidelines in the near future.
\end{abstract}

\section{Subject Areas \\ Health Policy}

\section{Keywords}

Good Medical Practice, Guidelines, Medical Students, Doctors 


\section{Introduction}

Good Medical Practice (GMP) are guidelines or professional code of practice which are primarily established as guidance for doctors in their respective countries [1]-[6]. It highlights the standards of care and professionalism, which is expected from doctors, as set out by their national medical councils [7]-[12]. Aside from exploring the moral, ethical and professional obligations expected from medical practitioners, these guidelines also aim to aid individual doctors in delivering good medical care and act as a supplemental resource to enhance the culture of medicine [8] [13]. Ultimately, the goal of having these guidelines is to protect the community by ensuring the provision of safe, effective and trustworthy medical services [14] [15].

In Malaysia, the Malaysian Medical Council (MMC) first issued the GMP guidelines in 2001 [16]. It was an 18-page booklet that discussed the 10 Golden Rules of GMP as well as the doctor's role in various settings. The guideline was subsequently revised in 2019 to incorporate new issues or aspects that have become more relevant over the years, as advised by the medical fraternity [7]. Similarly, Australia also issued a code of conduct for their doctors under section 39 of the Health Practitioner Regulation National Law. The original code was developed by the Australian Medical Council (AMC) together with some stakeholders. The Medical Board of Australia later adopted this code in 2010 after making minor revisions to ensure the consistency with national law [17] [18]. The latest, revised edition of the code was issued in 2020 [8].

Moreover, GMP guidelines are also issued in the United Kingdom (UK) by the General Medical Council (GMC), which was, in fact, the earliest among the three countries to do so. In the UK, GMP was first published in 1995 as a series of booklets under the "Duties of a Doctor" general heading [19]. Currently, GMC has two different guideline booklets to cater for doctors and medical students in the UK. The current guidance for medical students was jointly published by GMC and Medical Schools Council (MSC) in 2016; it highlights how GMP applies to medical students, and the expectations set on them. On the other hand, guidance for doctors was published in 2013, with the latest update being made in November 2020 [20].

GMP guidelines play an essential role in guiding medical professionals and ensuring provision of high-quality care to patients, as it touches on various key aspects which directly and indirectly influences patient outcome [21] [22] [23]. To date, many countries worldwide have produced GMP guidelines to regulate medical practice in their local setting [24]-[31]. The implementation is often overseen by their respective medical councils. These guidelines are regularly updated in line with the changing relationships of the profession with patients and general society.

While fundamental objectives and values may be similar across different country-based guidelines, differences might exist in the standards of competence, care and conduct expected across countries. In this review paper, we analyzed the GMP guidelines from three countries, including Malaysia, Australia 
and the UK to identify the key themes, similarities, differences and gaps in the existing guidelines. This subsequently enables the compilation of a more comprehensive GMP guideline for doctors and medical students. The findings discussed in this review will also facilitate policymakers to make the necessary correction while updating the respective guidelines in the near future.

\section{Comparison of the GMP Guidelines of Malaysia, Australia and the UK}

In this review paper, we compared the three country-specific (Malaysia, Australia and the UK) GMP guidelines under four domains: Domain-1 "Knowledge, skills and performance", Domain-2 "Safety and Quality", Domain-3 "Communication, partnership and teamwork" and Domain-4 "Maintaining trust". The thematic framework of domain 1 and 2 is presented in Figure 1, and that of the domain 3 and 4 is presented in Figure 2.

\subsection{Domain 1: Knowledge, Skills and Performance, Which Is Categorized under Three Sub-Domains}

\subsubsection{Develop and Maintain Professional Performance}

"Be competent in all aspects of work by keeping professional knowledge

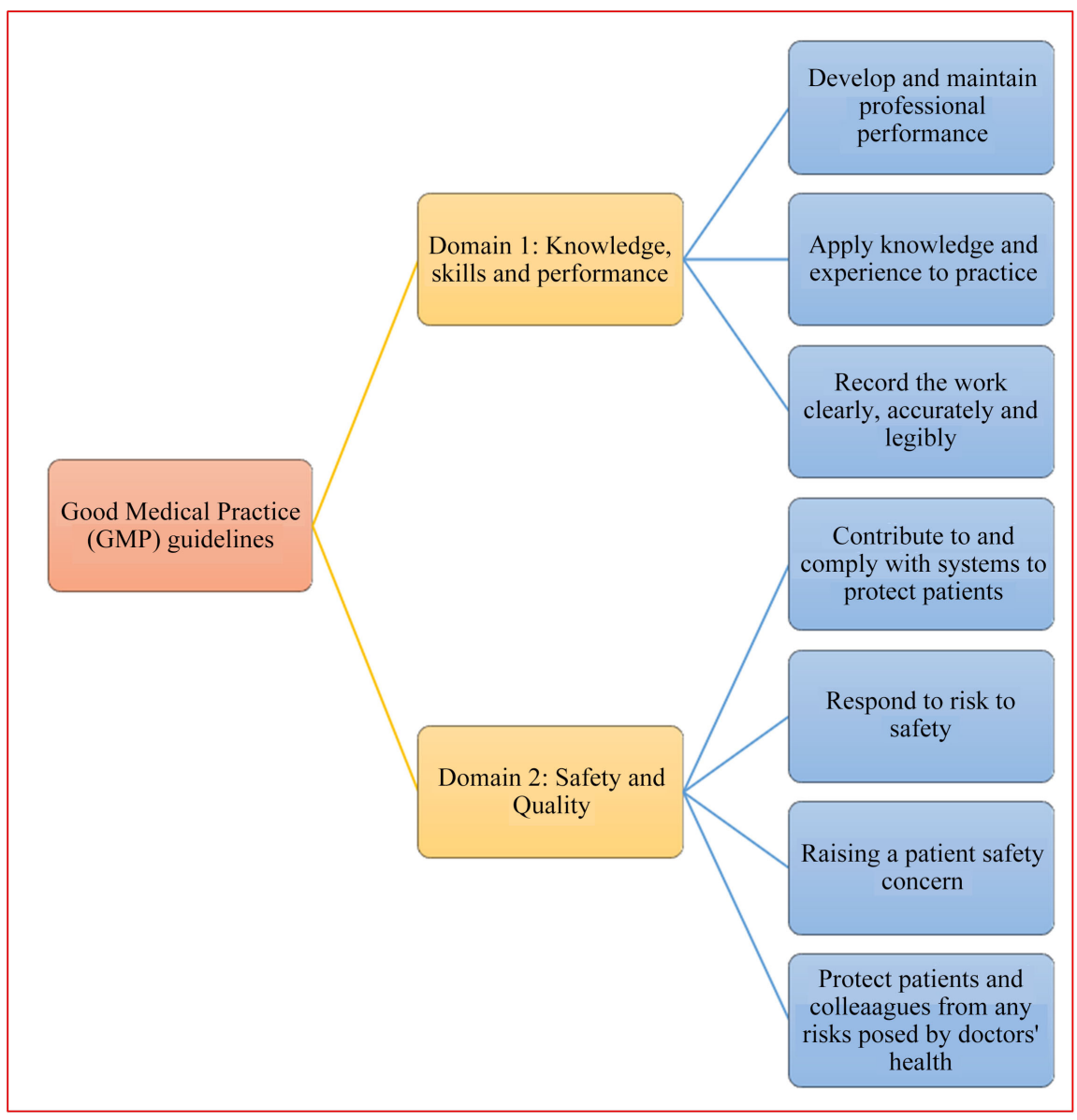

Figure 1. Thematic framework generated for subthemes under domain 1 and domain 2. 


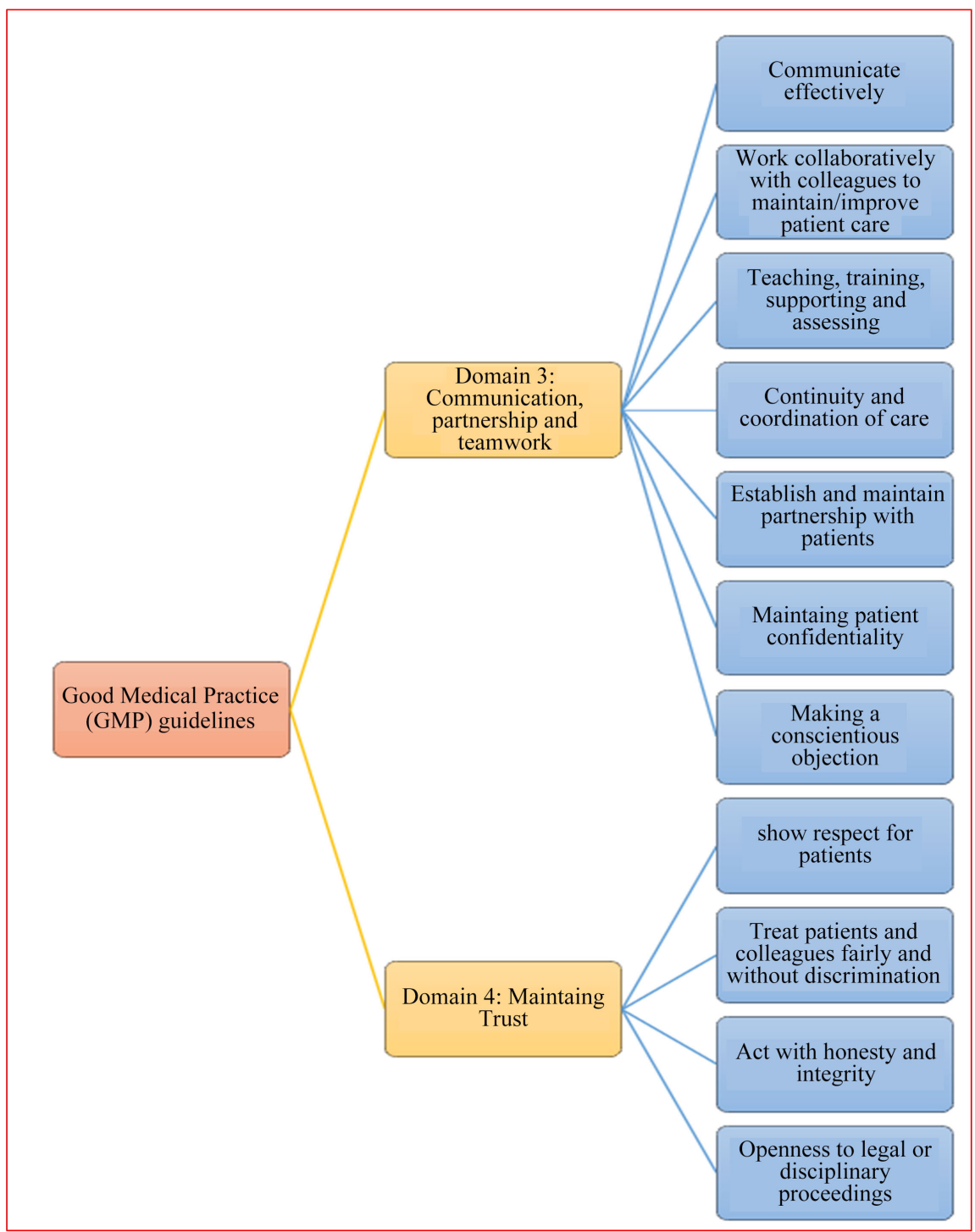

Figure 2. Thematic framework generated for subthemes under domain 3 and domain 4 .

and skills up-to-date and participating in activities to develop competence": Under this sub-domain, the respective medical councils of the UK, Australia and Malaysia emphasized that doctors and medical students should take active measures to keep their knowledge up-to-date and improve their skills as expected of their profession. It is a continuous and lifelong process to ensure high competence leading to better performance and patient care. Continuing Medical Education (CME) is now a standard practice in Malaysia, whereby a minimum number of Continuing Professional Development (CPD) points is now required for renewal of the Annual Practicing Certificate (APC) to practice in Malaysia. Similarly, regular planning and review of CPD activities are also encouraged in Australia to ensure that they are relevant to the physician's practice and fall in line with the Medical Board's requirement. In the UK, GMC has developed a guidance booklet on Continuing Professional Development for all doctors [32]. This booklet highlights various aspects of $\mathrm{CPD}$, ranging from planning to eva- 
luating the impact and ensuring the quality of activities participated by the doctors.

"Be familiar with guidelines and adhere to law and regulations": It is imperative that doctors must practise within moral, legal and ethical norms that have been deemed appropriate and constantly regulate themselves to uphold it, as highlighted in the Malaysian GMP guidelines. Moreover, the UK guidelines also emphasized the importance of proactively seeking information about policies or procedures that are relevant to their clinical placements. Doctors and medical students are also required to comply with all laws and regulations that have been put in place by the medical councils or institutions.

"Monitor and improve quality of work": Doctors are encouraged to engage in regular reflection on their practice and outcomes. Feedbacks and advice should be received with an open heart, critically reflected upon and used as a guide to constructing action plans to improve the quality of work. These aspects are pointed out in both the UK and Australia GMP guidelines.

\subsubsection{Apply Knowledge and Experience to Practice}

"Work within limits of the competence": Doctors should recognise their professional limitations and seek help/opinion whenever necessary, as pointed out in all three country guidelines. They must work within their scope of practice and utilise the best available information when suggesting treatment options. Additionally, the UK and Australian guidelines also highlighted that involvement of medical students in the patients' care should be made clear from the very beginning. Students should only attempt to commence treatment, consultation or practical procedures under appropriate supervision or with prior training.

"Provide good standard of practice and care": Patients should be adequately assessed at all stages prior to commencing a suitable management plan. This practice includes appropriate investigations, treatment and referral if necessary. Advice should be sought out from more experienced colleagues when the need arises, as emphasized in the UK and Australia guidelines. Moreover, it is crucial for doctors to not issue prescriptions before examining patients. Any adverse reactions to previous medications should be explored, and patients should be informed of the medications' purposes. Medications should only be prescribed for an appropriate convenient duration, particularly for diseases that require periodic monitoring. As for repeat prescriptions, the procedures for generating them should be secure, and patients' adherence should be discussed during each review. This aspect of prescribing is discussed thoroughly in the Malaysian guideline.

"Avoid providing medical care to themselves or anyone with whom they have a close personal relationship": As pointed out in all three country guidelines, doctors should avoid providing medical care or carrying out procedures on those with whom they have a close personal relationship. The Australian guidelines specifically mentioned that this practice also includes prescription of psy- 
chotropic medications, drugs of dependence and performing elective surgeries. In cases where the provision of care is necessary and unavoidable, the doctor should consider and manage these issues carefully.

"Informed consent before any procedure": Doctors must be professional in their conduct and appearance at all times. This practice includes physical appearance, manners, behaviour, and general composure. Identification badge or equivalent identification should be visible always, as emphasized in the UK and Malaysian guidelines. Prior to obtaining consent, complete information should be provided and explained in a way that guarantees the patient's understanding, and in the case of major invasive procedures, possible postoperative complications should be explored. It is also highlighted in the Australian guideline that patients should be advised regarding the fees, charges and possible additional costs in a timely manner. Any refusals should be noted in the patients' records. Patients' decisions should be respected, and no procedures should be carried out without their consent. Consent should also be obtained prior to any recordings or involvement in teaching and research activities.

In some cases, patients may not have the capacity to consent, and decisions will be made by substitute decision-maker or by considering legal documents such as advance care directives. However, if there are no relatives present to give consent and emergency lifesaving intervention is required, the attending doctor and another registered medical practitioner may manage the patient in the patient's best interest. If the patient is a minor, informed consent should be obtained from parents, next-of-kin or legal guardian. The involvement of medical students should be informed to the patients, and consent should be obtained as well. This aspect was explored in the UK and Australian guidelines.

"Engage in routine evaluation systems": Doctors should engage in professional development and appraisal processes relevant to their roles, as discussed in the UK and Australian guidelines. Additionally, the UK guidelines also explored the role of medical students, whereby they are expected to engage in routine evaluation systems, attend induction and all mandatory training sessions. It is also crucial for students to recognise their direct supervisors and person-in-charge to ease the process of raising concerns and seeking clarification about placement-related issues.

"Make good use of available resources": Responsible and effective use of available resources is vital for doctors as it can affect other patients' access to healthcare. This is emphasized in the Malaysian and Australian guidelines. Doctors must be mindful of the heightening cost of healthcare provision and delivery and take steps to preserve high quality despite restrictions. Patients' right to access necessary healthcare services should be upheld at all times, together with transparent and equitable allocation of resources. Furthermore, the Malaysian guideline also highlighted that the disposal of clinical wastes and toxic by-products of medical practice should be handled in the least harmful manner as possible. 


\subsubsection{Record the Work Clearly, Accurately and Legibly}

"Recording of work must be clear, legible and accurate": Records must be clear, accurate, legible, comprehensive, honest, up-to-date and non-judgemental. They should aid in easy understanding and recall. Malaysian and Australian guidelines also specified that recorded notes should not be erased or altered. Instead, errors should be neatly deleted but still readable, and changes or additions made should always be dated. Proper stock inventory should be maintained for all medications in the facility, including dangerous and controlled drugs, as highlighted in the Malaysian guideline. Moreover, the UK guidelines also emphasized that medical students should employ a professional manner of writing for documentation submitted to the school. Notes made as part of the learning process should be clear, accurate and legible as well.

"Record as soon as possible": Notes should be recorded at the time of event or as soon as possible afterwards, including after interaction with patients. This was discussed in both Australian and the UK guidelines.

"Secure storage of records, in line with any data protection law requirements": All three country guidelines highlighted the importance of secure storage of records. While clinical notes and records are physically kept with the doctor or healthcare institution, the information contained still belongs to the patients or regulatory authorities as demanded by law. Hence, medical records and confidential material should be stored securely and protected against unauthorised access. They should be retained for periods required by law before being disposed of securely, as pointed out in the Australian and UK guideline. Furthermore, proper records and secure storage are also essential in handling of dangerous and controlled drugs and this was discussed in the Malaysian guideline.

"Records should include the following content": Records should include relevant details of clinical history, findings, investigation, diagnosis, decisions made and actions agreed upon, the information provided, medications prescribed, referrals and other management information. As for invasive procedures, indications and nature of procedures must be documented. All medical records should be written clearly in an appropriate detail, including the date, time and name of the attending doctor. Malaysian guidelines also specified that records of prescription for dangerous and controlled drugs should be stored with treatment details. Medical students should indicate their status in the notes as well, as highlighted in the UK guidelines. Importantly, the content should always be written in a manner that reflects appropriate respect for patients, and this aspect was noted in the Australian guideline.

\subsection{Domain 2: Safety and Quality}

\subsubsection{Contribute to and Comply with Systems to Protect Patients}

"Participate in quality assurance and quality improvement activities; reflect on standards of care delivered; review and provide constructive feedbacks": These aspects are highlighted in the UK and Australian guidelines. 
Doctors should take part in systems of quality assurance and improvements such as audits and regular reviews, and surveillance and monitoring of adverse events and "near misses". They must always seek to enhance the quality of services delivered at the individual and systems level. Systems should be set in place to enable raising concerns about risks to patients. Measures should also be taken to reduce the risk of fatigue by working safe hours wherever possible and adhering to relevant occupational health and safety legislation, wherever available. Complaints received should be reflected and acted upon.

"Keep patient safe-report adverse events": It was emphasized in the Australian guideline that all doctors are responsible for clinical governance and should acknowledge the importance of an open-disclosure and a non-punitive incident management approach. Immediate actions should be taken to rectify problems in light of any adverse events, and relevant policies, procedures, as well as reporting requirements which should be adhered to. These events should be reviewed and reflected upon to implement changes that may subsequently reduce recurrence risk. The UK guidelines also specified that medical students may be asked to contribute to internal inquiries, and they should engage honestly and openly in the process.

"Respond to requests/assist in public health monitoring": Doctors should utilise their influence and expertise to address healthcare inequity and advance general health and well-being. They should also recognise their obligations in disease prevention, screening, and reporting of notifiable diseases, aside from involving themselves in efforts to promote the health of the community. This aspect was highlighted in the Australian guideline.

\subsubsection{Respond to Risk to Safety}

"Promote a culture that allows all staff to raise concerns openly, take prompt action if patient safety is compromised": Patient safety includes raising concerns when a patient's dignity or comfort is compromised. This aspect is addressed in all three country guidelines. It is crucial for doctors to reduce errors, improve patient safety and provide adequate support for colleagues who raised concerns on patient safety in their practice. In the interests of the public, it is also a doctor's duty to highlight their colleague's practice to relevant higher authorities if it has the potential to compromise patients in any way. Medical students should follow their school's policy in raising concerns, wherever possible. Additionally, the Malaysian guideline also specified that in terms of facilities and services, the general appearance of clinic and consultation rooms should appeal to the patients by providing a calm, soothing and reassuring ambience. Cleanliness and neatness should be maintained, and clinical equipment should be in good working order. Appropriate behaviour and mannerism of staff are also important.

"Offer help if emergencies arise": Doctors are obligated to provide emergency or lifesaving treatments to patients regardless of social and financial status, or suspicion of serious communicable diseases (when the doctor and staff 
take the standard precautions). They should offer assistance in an emergency while considering their safety, skills and availability of other options. Provision of assistance should be continued until the service is no longer required. This is emphasized in Malaysian and Australian guidelines.

"Ensure rights of vulnerable adults or children are not compromised":

The Australian guideline also noted that it is important for doctors to be alert to children and young people who may be at risk and notify relevant authorities where appropriate. Abuse of wives, the elderly and other vulnerable population groups could be brought to light by doctors as well.

\subsection{3. "Raising a Patient Safety Concern"}

Doctors should raise a genuine concern about patient safety to relevant authorities and adhere to mandatory reporting requirements, as highlighted in the UK and Australian guidelines. They should not make vexatious complaints about their colleagues. In fact, concerns should be raised in a confidential, nonjudgemental manner. They should also ensure that they have gathered the correct facts about a situation prior to raising concern and seek alternative methods of raising concerns if the conventional manner is not suitable or comfortable in the specific circumstance. Medical students also have a moral responsibility to raise concerns about patient safety, dignity and comfort, as noted in the UK guideline. They may refer to placement provider's raising concerns policy or consult their school or experienced healthcare professional for advice. Avoid raising concerns anonymously wherever possible as organisations may find it more difficult to investigate anonymous complaints.

\subsubsection{Protect Patients and Colleagues from Any Risk Posed by Doctors' Health}

"Ask advice from colleague and make necessary changes when suspecting that doctors have a serious health condition": It was highlighted in all three country guidelines that doctors must protect patients from any risk posed by their health and seek independent, objective advice from a suitable colleague. They must not rely on their own assessment but should consult their doctor for advice and help to modify their practice as necessary. They must also endeavour to work safe hours whenever possible. Medical students should follow their school's guidance and obtain appropriate support, as emphasized in the UK guideline. In cases of stress or anxiety, concerns should be communicated with General Practitioners (GP) or through other appropriate sources such as helplines.

"Informing when doctors have a condition": Any health conditions which may limit fitness to practice should be declared to relevant authorities. This aspect is covered in both UK and Australian guidelines. Doctors should be aware of and comply with these reporting obligations. The advice can also be sought from a professional indemnity insurer in the case of uncertainties. As for medical students, they should also inform their school about any aspect of health or personal circumstances that may interfere with training or relationships with 
colleagues.

"Getting immunised": Doctors should ensure that they are immunised against relevant communicable diseases, as noted in the UK and Australian guidelines. Students should also adhere to the occupational health policies and procedures of their institution. They should engage with occupational health referral processes in the case of deterioration of health. Moreover, students with blood-borne viruses must complete recommended health screening and may be required to practice restrictions in their clinical placements and medical practice upon graduation.

"Getting independent medical advice": All three country guidelines emphasized that doctors should be aware of the risks posed by self-diagnosis and self-treatment. They should seek independent, objective medical advice and avoid treating themselves. This practice applies to stress, burnout, anxiety and depression as well. Students may seek advice from their GP or other appropriately qualified healthcare professional. Treatment plans prescribed by the attending doctor should be adhered to, and changes should not be made prior to consulting them.

"Registering/having access to a general practitioner": It is imperative that doctors and medical students have access to general practitioners whom they may consult for issues or queries related to their health. This aspect is highlighted in the UK and Australian guidelines.

\subsection{Domain 3: Communication, Partnership and Teamwork}

\subsubsection{Communicate Effectively}

"Listen to patients' views": In a doctor-patient partnership, doctors should encourage patients to voice their opinions and concerns, confirm patients' understanding, listen to and respect their perspectives on their health in a decision-making process, as noted in all three country guidelines. This aspect is also discussed extensively in the Malaysian guideline, whereby it was mentioned that a doctor should also be attentive, patient and compassionate during consultations, without hurrying or patronising patients.

"Be open and honest": The UK and Australian guidelines also discussed that doctors should be open and honest while communicating with patients, experiencing uncertainties and reporting adverse events. A thorough assessment of patients' character is also necessary to ensure smooth communication as patients may present from different backgrounds. Malaysian guidelines specified that doctors should avoid presenting themselves as the ultimate healer. Instead, they should try their best to seek answers, either by themselves or with others' help.

"Be readily accessible with information, advice and support tailored to patients' wants or needs": Doctors should be polite and considerate to patients and, if present, their concerned family members at all times. Practical measures should be taken to address any specific language, culture, communication or other potential barriers to ensure patients' understanding. This practice is covered in all three guidelines and is deemed important as lack of communication 
and anger from the doctors' side may lead to negative adverse outcomes such as lawsuits. Patients should also be informed about their condition and all available management options, including its potential risks and benefits. The Malaysian guideline also highlighted that doctors should periodically explain the need for a particular step during the physical assessment to help patients relax and cooperate. Any discrepancies between the doctor and patient should be resolved together through diplomacy without belittling the patient.

\subsubsection{Work Collaboratively with Colleagues to Maintain/Improve Patient Care}

"Work collaboratively with colleagues": Doctors should acknowledge, communicate effectively and work collaboratively with all healthcare professionals as a team. Taking into consideration their own limitations, doctors should inform and refer patients to a specialist colleague for the best treatment available, as highlighted in the Malaysian guideline. They should also seek an independent opinion from colleagues if they find their clinical judgement compromised by the organisational culture. Moreover, the Australian guideline also discussed about the importance of advocating for a clear delineation of roles and responsibilities among team members and informing patients regarding it.

"Respect and treat colleagues fairly": Doctors should behave professionally, respectfully and courteously to all colleagues. In the Malaysian guideline, it was mentioned that they should avoid criticising or demeaning their colleagues, especially in the presence of a patient. The referred doctor should also obtain consent from the primary doctor before further referring patients to a third specialist. Both the Malaysian and Australian guidelines highlighted a doctor's role in ensuring a learning environment free from discrimination, bullying and harassments for their junior colleagues. Additionally, the Australian guideline also touched on the importance of professional behaviour towards colleagues and other practitioners while using social media.

"Be aware of the impact of own behaviour on others": Understanding the impact of their behaviour on the team's performance helps doctors to adapt to achieve team goals easily. They should be tolerant, patient and possess behaviour that reflects themselves as positive role models. Avoid seeing colleagues as competitors or rivals. This aspect is discussed in all three country guidelines.

\subsubsection{Teaching, Training, Supporting and Assessing}

"Be prepared to teach and train others": Doctors should be available and willing to train their junior colleagues. The Australian guideline also highlighted the need to develop effective teaching skills actively.

"Be honest and objective in referencing, appraising or assessing others": Doctors should provide adequate supervision and constructive feedback for the assessment of doctors in training or appraisals of colleagues in an honest, objective and respectful manner. They should be accurate and evidence-based. This section, however was not included in the Malaysian guideline. 
"Support colleagues with their performance and health": Doctors should support their colleagues who are unwell or underperforming by providing them with the same quality of care, encouraging them to seek appropriate help or reporting to the relevant bodies. This aspect is covered in the Australian guideline.

\subsubsection{Continuity and Coordination of Care}

"Safe transfer": Whenever they are unavailable, doctors should provide advance notice and ensure continuity of care. Safe transfer of patients includes coordinating efficient handover procedures as well as delegating and sharing relevant information to someone who has the qualifications, experience, knowledge and skills to care for the patient. In the Malaysian and Australian guidelines, the primary doctor remains responsible for the overall management of the patient and family members, but not accountable for any decisions made by doctors whom they delegated the patients to.

\subsubsection{Establish and Maintain Partnerships with Patients}

"Respect patients' dignity, privacy, life choices and beliefs": In a doctor-patient partnership, doctors should be polite, fair and considerate, regardless of their perspectives on the patients' life choices or beliefs. They should respect patients' queries, rights in decision-making, dignity and confidentiality. The Malaysian guideline also highlighted the role of a chaperone during a physical examination to ensure privacy. Additionally, the importance of treating the friends and family members of patients with courtesy and respect is also discussed.

"Share relevant information": Patients should be informed of their health, purpose, expected outcomes, possible complications and other available options before a procedure, investigation or treatment. Patients should not be coerced in a decision-making process, as highlighted in the Malaysian guidelines.

"Conscientious objection": Patients' right to seek a second opinion from a suitably experienced doctor should be respected. Doctors should make necessary arrangements and provide all relevant information and investigation results to avoid inaccurate clinical judgement by the referred doctor. This aspect is emphasized in the Malaysian and Australian guidelines.

\subsubsection{Maintaining Patient Confidentiality}

"Preserve confidentiality": As highlighted in all three country guidelines, doctors should keep all patient information confidential. However, confidentiality is not absolute. Doctors can disclose relevant information to the patient, next-of-kin or a third party with the patient's consent. In the Malaysian guideline, it was mentioned that explicit written consent from the patient or next-of-kin is required prior to entertaining any request for disclosure from third parties. Furthermore, doctors should also keep patients informed after a disclosure to legal or statutory requirements, consistent with privacy laws and professional guidelines.

"Avoid unintentional improper disclosures": Doctors should not share 
identifiable information in public places, social media, medical conferences, publications or logbooks. This is highlighted in the UK and Malaysian guidelines. Doctors should be aware of confidential information when discussing care in the presence of patient's friends and family members. The UK guideline also emphasized on the social media dos and don'ts when it comes to sharing information related to their practice.

"Discuss their patients": As highlighted in the UK and Malaysian guideline, all patient-related discussions should be conducted in a confidential manner only with the relevant parties. Except for the clinician directly involved in a patient's care, doctors should not discuss their patients with colleagues or supervisors in public. Discussion should remain factual without identifiable information.

"Store information confidentially": Doctors should, by all means, preserve privacy and confidentiality when storing and disposing of patients' information. This section is discussed in the UK and Malaysian guidelines.

\subsubsection{Making a Conscientious Objection}

Doctors should opt-out if they are uncomfortable providing certain treatment due to personal beliefs and values. They should carefully explain this to patients and make the necessary referrals without impeding access to treatment, discriminating or expressing disapproval of the patients' lifestyle, choices or beliefs. This aspect is also emphasized in all three country guidelines.

\subsection{Domain 4: Maintaining Trust}

\subsubsection{Show Respect for Patients}

"Must not use professional position to pursue personal relationship": All three country guidelines emphasized that doctors must respect their patients. They should recognise and maintain their professional boundaries by avoiding the development of private and personal relationships with their patients. They should not utilise their professional position to pursue any improper relationships with patients from physical, emotional, sexual and financial perspectives.

"Must not express personal beliefs in ways that exploit patients' vulnerability or cause distress": Doctors must not cause distress or exploit their patient's vulnerability by expressing their personal beliefs. In circumstances whereby a patient's own behaviour led to the development of their condition, the Malaysian guidelines emphasise doctors to treat patients appropriately without allowing personal views on a patient's lifestyle to influence their management. For instance, a doctor may believe that treating smokers with heart disease requiring an angiogram is a waste of time; however, this type of belief should be avoided, and it should never impact the provision of care to the patient. This is also mentioned in the Australian guideline.

"Be open and honest if things go wrong": The Australian and UK guidelines discussed the importance of being honest in the event something goes wrong. Doctors need to be open and explain to the patients the adverse events that have 
taken place promptly. In the UK, medical students are also required to report to their supervisors if they encounter any aspect of a patient's care that is inappropriate so that improvements can be made. In Australia, responding to patients after an adverse event is also discussed, for instance supporting and providing them adequate information if a complaint is to be filed. After a complaint is made, it should not negatively impact a patient's care, and it may be appropriate to refer them to another practitioner should there be doubts about maintaining the quality of care.

\subsubsection{Treat Patients and Colleagues Fairly and without Discrimination}

"Give patients the treatment they need based on clinical judgement": Before ordering investigations or initiating treatment, doctors must examine and evaluate the patient's need for them and their likely effectiveness. In Malaysia, the guideline also discussed about informing patients of the estimates of professional charges and avoiding fragmenting patient care by referring them to multiple specialists as it may increase a patient's financial expenses.

"Don't let personal views discriminate patients/colleagues": Doctors must treat everyone equally, including patients and colleagues, regardless of their social status, gender, sexual orientation, race, religion and disability. They must also report to relevant authorities should they encounter any form of discrimination. The UK guideline also touched on the importance of reflective practice to identify personal biases and response to people or certain situations.

"Respond promptly to feedback, apologise, provide insurance and indemnity cover": Doctors should act immediately by providing feedback if the patient is dissatisfied with the care provided. They should also express empathy by comforting the patients. It is a good practice for doctors to obtain professional indemnity cover for their practice. In Malaysia, this is a necessary requirement for private practitioners, while the Malaysian government covers government doctors. In fact, annual indemnity cover is mandatory for the issuance of APC.

"End a professional relationship with a patient only when there is a breakdown of trust": The UK guidelines also specifically discussed that a professional relationship should only end when trust has been broken.

\subsubsection{Act with Honesty and Integrity}

"Honest about experience": Doctors have to be honest and accurate about their experience and medical qualifications. Acts like self-promoting should not be practised. In the UK, medical students must not submit applications or coursework with misleading information and only sign in for teaching sessions that they have attended.

"Follow guidelines when designing and carrying out research": When carrying out research, it is imperative to follow the guidelines after obtaining approval from relevant authorities to ensure ethical requirements are fulfilled before initiating the project. For instance, disclosing the purpose, methods, risks and benefits. This practice ensures that participants provide informed consent to 
join the research. Should there be any misconduct, the doctors must report to the appropriate committee. This aspect is heavily emphasized in the Malaysian and Australian guidelines.

"Maintain confidentiality on social media": Doctors should utilise social media ethically and legally to avoid reputational damage and preserve patients' confidentiality by not disclosing identifiable information. In the UK, GMC has also produced a specific guideline concerning the use of social media by doctors [33].

"Accurate advertisement information": It is also good practice to have verifiable advertisement information, for example, the quality and outcome of the services provided. There should not be imprecise comparisons occurring among colleagues, as highlighted in the Australian guideline. Moreover, the Malaysian guideline also focused on the information that should be available in the name card. When participating in charitable events, it should not be utilized as a tool to increase publicity.

"Honest when writing reports and academic writing": Doctors should also display honesty in writing, irrespective of whether it is an academic document or report. They should not submit any material constituting plagiarism or falsify results while carrying out research. In terms of medical reports or certificates, both Australia and Malaysia demonstrated that doctors should gather sufficient and accurate information before certifying these documents.

\subsubsection{Openness to Legal or Disciplinary Proceedings}

\section{1) Honest when giving evidence}

"Cooperate with formal inquiries and complaint procedures": As highlighted in all three country guidelines, doctors should also be honest when providing evidence in legal or disciplinary proceedings and cooperate with a formal enquiry as well as complaint procedures without withholding any relevant information. Doctors must work together with patients in resolving the issue that led to a complaint.

2) Understand the limits of competence when giving evidence

"Report if receive a caution from police or charged": While giving evidence, doctors should also recognise their own limits of competence by not providing information beyond their own capabilities. The Malaysian and Australian guidelines did not discuss this aspect but the UK guidelines specified the duty of a doctor and medical student to report to the institution or GMC when they have been cautioned or charged by police to determine their fitness to practice medicine.

"Honesty in financial dealings": In financial dealings, doctors must display honesty by making patients aware of the estimated cost of treatment and charging reasonably by providing necessary services and not exploiting vulnerable patients with a lack of medical knowledge. When patients personally demand a service that may be unnecessary, doctors should gently educate them and correct any inaccurate concepts. When patients are in financial difficulty, and the cost of 
treatment may increase their burden, it is wise for doctors to transfer them to public or less costly private hospitals to ensure continuity of care. This aspect is covered in the Malaysian guideline. Moreover, the Australian guideline also stated that doctors should also be transparent in all financial dealings and avoid involving financially with patients, for instance through loans or investment schemes.

"Not ask for or accept inducements or gifts": Doctors should not request for or accept inducements of any forms for their own benefit directly or indirectly, as highlighted in the Malaysian and Australian guideline. Examples include when referring patients to other doctors or from pharmaceutical companies. When doctors are offered attractive gifts in return for attending conferences to promote pharmaceutical products or equipment, they should carefully evaluate the motives. These offers should also be made through healthcare organisations.

\section{Conclusions}

In summary, Good Medical Practice (GMP) guidelines are implemented in many countries worldwide and are primarily regulated by their respective medical councils. In this review, we have looked into GMP guidelines from three different countries and identified the core themes that have been incorporated into them. While there are huge similarities in the content of the guidelines, variations do exist in terms of subthemes and depth of discussion for certain aspects.

Based on the results of our review, several recommendations that could be made include firstly, to come up with a standardised document to record doctors' immunisation status. Aside from serving as a guide for doctors to advise them on necessary immunisation requirements as per the national guidelines, it would also enable standardised monitoring of doctors' immunisation status by relevant authorities. Secondly, formal measures to reduce fatigue among doctors should be undertaken in all countries through occupational health and safety legislation, as currently, the focus is on doctors to try and work safe hours where possible, which might lead to discrepancies in workload across departments, hospitals and states.

GMP guidelines undoubtedly play a crucial role in guiding medical professionals to provide a high quality of care to patients. This review serves as a comprehensive compilation of GMP guidelines to be utilized by doctors and medical students in their daily practice. As we consider the constant changes in doctor-patient relationships and general society in the current era, it is imperative that these guidelines be reviewed and updated regularly in line with recent changes and developments in the medical professional society.

\section{Acknowledgements}

Authors would like to acknowledge Monash University for providing permission to utilize the extensive resources from Monash University Malaysia library, in- 
cluding access to all the required full-text articles. Moreover, Monash University also provided Endnote software which was utilized by the authors to remove duplicated articles and to manage the references, and Nvivo software for data extraction.

\section{Conflicts of Interest}

The authors declare no conflicts of interest regarding the publication of this paper.

\section{References}

[1] Gill, D. and Griffin, A. (2010) Good Medical Practice: What Are We Trying to Say? Textual Analysis Using Tag Clouds. Medical Education, 44, 316-322. https://doi.org/10.1111/j.1365-2923.2009.03588.x

[2] Gillon, R. (2015) Defending the Four Principles Approach as a Good Basis for Good Medical Practice and Therefore for Good Medical Ethics. Journal of Medical Ethics, 41, 111. https://doi.org/10.1136/medethics-2014-102282

[3] Palmer, K.T., Harling, C.C., Harrison, J., Macdonald, E.B. and Snashall, D.C. (2002) Good Medical Practice: Guidance for Occupational Physicians. Occupational Medicine, 52, 341-352. https://doi.org/10.1093/occmed/52.6.341

[4] Hutchinson, A., Williams, M., Meadows, K., Barbour, R.S. and Jones, R. (1999) Perceptions of Good Medical Practice in the NHS: A Survey of Senior Health Professionals. Quality in Health Care, 8, 213. https://doi.org/10.1136/qshc.8.4.213

[5] Jaques, H. (2013) GMC Publishes New Version of Good Medical Practice. BMJ, 346, f2003. https://doi.org/10.1136/bmj.f2003

[6] Gough, I.R. (2014) The Significance of Good Medical Practice: A Code of Conduct for Doctors in Australia. Medical Journal of Australia, 200, 148-149. https://doi.org/10.5694/mja13.11334

[7] Malaysian Medical Council (2019) Good Medical Practice 2019. 43.

[8] Medical Board of Australia (2020) Good Medical Practice: A Code of Conduct for Doctors in Australia. 27.

[9] United Kingdom: General Medical Council (GMC), Medical Schools Council (MSC) (2016) Achieving Good Medical Practice: Guidance for Medical Students. 64.

[10] Flynn, J.M. (2008) Good Medical Practice: Developing an Australian Code. Medical Journal of Australia, 189, 477-478. https://doi.org/10.5694/j.1326-5377.2008.tb02137.x

[11] Irvine Sir, D. (2006) A Short History of the General Medical Council. Medical Education, 40, 202-211. https://doi.org/10.1111/j.1365-2929.2006.02397.x

[12] Irvine, D. (2001) Doctors in the UK: Their New Professionalism and Its Regulatory Framework. The Lancet, 358, 1807-1810. https://doi.org/10.1016/S0140-6736(01)06800-3

[13] Rubin, P. and Franchi-Christopher, D. (2002) New Edition of Tomorrow's Doctors. Medical Teacher, 24, 368-369. https://doi.org/10.1080/0142159021000000816

[14] Gill, D. and Griffin, A. (2010) Good Medical Practice: What Are We Trying to Say? Textual Analysis Using Tag Clouds. Medical Education, 44, 316-322. https://doi.org/10.1111/j.1365-2923.2009.03588.x 
[15] Cowan, J. (2007) Good Medical Practice Should Improve Patient Safety. Clinical Governance: An International Journal, 12, 136-141. https://doi.org/10.1108/14777270710741492

[16] Malaysian Medical Council (2001) Good Medical Practice. 18.

[17] Australian Medical Council Limited (2009) Good Medical Practice: A Code of Conduct for Doctors in Australia. 40.

[18] Medical Board of Australia (2010) Good Medical Practice: A Code of Conduct for Doctors in Australia. 22.

[19] McManus, I.C., Gordon, D. and Winder, B.C. (2000) Duties of a Doctor: UK Doctors and Good Medical Practice. Quality in Health Care, 9, 14-22. https://doi.org/10.1136/qhc.9.1.14

[20] Manchester: General Medical Council (2013) Good Medical Practice. 38.

[21] Polonsky, W.H., Capehorn, M., Belton, A., Down, S., Alzaid, A., Gamerman, V., et al. (2017) Physician-Patient Communication at Diagnosis of Type 2 Diabetes and Its Links to Patient Outcomes: New Results from the Global IntroDia ${ }^{\circledR}$ Study. Diabetes Research and Clinical Practice, 127, 265-274. https://doi.org/10.1016/j.diabres.2017.03.016

[22] Baggs, J.G., Schmitt, M.H., Mushlin, A.I., Mitchell, P.H., Eldredge, D.H., Oakes, D., et al. (1999) Association between Nurse-Physician Collaboration and Patient Outcomes in Three Intensive Care Units. Critical Care Medicine, 27, 1991-1998. https://doi.org/10.1097/00003246-199909000-00045

[23] Arora, N.K. (2003) Interacting with Cancer Patients: The Significance of Physicians' Communication Behavior. Social Science \& Medicine, 57, 791-806. https://doi.org/10.1016/S0277-9536(02)00449-5

[24] Laranjo, L., Quiroz, J.C., Tong, H.L., Arevalo Bazalar, M. and Coiera, E. (2020) A Mobile Social Networking App for Weight Management and Physical Activity Promotion: Results From an Experimental Mixed Methods Study. Journal of Medical Internet Research, 22, e19991. https://doi.org/10.2196/19991

[25] New Delhi: Medical Council of India (2002) (Professional Conduct, Etiquette and Ethics) Regulations, 2002. 15.

[26] Republic of Ireland: Medical Council of Ireland (2009) Guide to Professional Conduct and Ethics for Registered Medical Practitioners. 33.

[27] Dubai: Dubai Health Authority (2014) Code of Ethics and Professional Conduct. 32.

[28] New Zealand: Medical Council of New Zealand (2016) Good Medical Practice. 38.

[29] Singapore: Singapore Medical Council (2016) Ethical Code and Ethical Guidelines. 65.

[30] Canada: Canadian Medical Association (2018) CMA Code of Ethics and Professionalism. 7.

[31] Sharman, M.J., Nash, M. and Cleland, V. (2019) Health and Broader Community Benefit of Parkrun-An Exploratory Qualitative Study. Health Promotion Journal of Australia, 30, 163-171. https://doi.org/10.1002/hpja.182

[32] Manchester: General Medical Council (2012) Continuing Professional Development: Guidance for All Doctors. 32.

[33] General Medical Council (GMC) (2013) Doctors' Use of Social Media. 4. 


\section{List of Abbreviation}

$\begin{array}{ll}\text { AMC } & \text { Australian Medical Council } \\ \text { APC } & \text { Annual Practicing Certificate } \\ \text { CME } & \text { Continuing Medical Education } \\ \text { CPD } & \text { Continuing Professional Development } \\ \text { GMC } & \text { General Medical Council } \\ \text { GMP } & \text { Good Medical Practice } \\ \text { GP } & \text { General Practitioners } \\ \text { ID } & \text { Identification } \\ \text { MMC } & \text { Malaysian Medical Council } \\ \text { MSC } & \text { Medical Schools Council } \\ \text { UK } & \text { United Kingdom }\end{array}$

